\title{
Fault Detection and Diagnosis System for a Three-Phase Inverter Using a DWT-Based Artificial Neural Network
}

\author{
Ali Rohan ${ }^{1}$ and Sung Ho Kim ${ }^{2}$ \\ ${ }^{1}$ School of Electronics and Information Engineering, Kunsan National University, Gusan, Korea \\ ${ }^{2}$ School of IT Information and Control Engineering, Kunsan National University, Gunsan, Korea
}

\section{]jfis}

\begin{abstract}
Inverters are considered the basic building blocks of industrial electrical drive systems that are widely used for various applications; however, the failure of electronic switches mainly affects the constancy of these inverters. For safe and reliable operation of an electrical drive system, faults in power electronic switches must be detected by an efficient system that is capable of identifying the type of faults. In this paper, an open switch fault identification technique for a three-phase inverter is presented. Single, double, and triple switching faults can be diagnosed using this method. The detection mechanism is based on stator current analysis. Discrete wavelet transform (DWT) using Daubechies is performed on the Clarke transformed (-) stator current and features are extracted from the wavelets. An artificial neural network is then used for the detection and identification of faults. To prove the feasibility of this method, a Simulink model of the DWT-based feature extraction scheme using a neural network for the proposed fault detection system in a three-phase inverter with an induction motor is briefly discussed with simulation results. The simulation results show that the designed system can detect faults quite efficiently, with the ability to differentiate between single and multiple switching faults.
\end{abstract}

Keywords: Stator current analysis, Discrete wavelet transform (DWT), Feature extraction, Fault detection and identification, Inverter switching faults, Variable speed AC drive, Induction motor, Artificial neural network system, Clarke transformation.

Received: Nov. 30, 2016

Revised : Dec. 12, 2016

Accepted: Dec. 13, 2016

Correspondence to: Sung Ho Kim

(shkim@kunsan.ac.kr)

(CThe Korean Institute of Intelligent Systems

(c) This is an Open Access article distributed under the terms of the Creative Commons Attribution Non-Commercial License (http://creativecommons.org/licenses/ by-nc/3.0// which permits unrestricted noncommercial use, distribution, and reproduction in any medium, provided the original work is properly cited.

\section{Introduction}

Motor drive systems that comprise induction machines and voltage source inverters (VSIs) are vulnerable to various faults in power inverters, induction machines, and control subsystems. In an industrial application, such faults in the system can lead to a complete shutdown of operation due to unexpected and long delays during maintenance schedules. Such maintenance can affect the overall cost of such systems, and because, there is a need to develop fault tolerant motor drive systems. In most industrial applications, VSIs are used for variable speed operation of induction motors; however, the usage of such inverters has some disadvantages that include a high probability of power electronic switches failure (IGBT, MOSFET, BJT). There are different types of switch failures. Open switch and short switch faults are among the most common, which lead to current distortion and degrade system performance. Various techniques presented in the past used inverter current [1, 2], voltages [3, 4], and FFT patterns for output voltage [5] to design fault detection systems. In another paper, wavelet decomposition using 
discrete wavelet transform was used to extract valuable features [6, 7].

In this paper, a fault detection and diagnosis system based on an artificial neural network is proposed by performing a discrete wavelet transform on Clarke transformed $(\alpha-\beta)$ stator current for feature extraction. The main advantage of the wavelet transform is the decomposition of electrical transients into a series of wavelet components. After that, identification and localization of the sources behind these transients performed using the wavelet components. In this study, the emergence of electrical transients during open and close switch faults in single, double, and triple switches in an inverter are decomposed using wavelet transform. Features like energy content and mean of the signals were extracted from the decomposed electrical transients. An artificial neural network technique was used for the detection and identification of switching faults based on those extracted features. A block diagram of this process is shown in Figure 1.

\section{Fault Detection and Diagnosis System}

The basic architecture of the fault detection and diagnosis system is shown in Figure 1. First, open switch faults in single and multiple switches were created through simulation and the feature extraction of the Clarke transformed current signals using discrete wavelet transformation analysis in MATLAB. Offline training of a neural network is performed in MATLAB using the extracted features.

\subsection{Clarke Transformation and Current Patterns}

Initially, the measured output current data of the induction motor is transformed from three phase to two phase using Clarke transformation. This transformation is performed for the evaluation of stator current patterns of an induction motor when switch faults occur in the inverter system as shown in Figure 2. Under normal conditions, the Clarke transformed current pattern resembles a circle whereas, in case of faults, the current pattern is biased to the direction of faulty switch with respect to missing phase.

\subsection{Discrete Wavelet Transform and Feature Extraction}

DWT techniques are known to be effective in the extraction of features from output current signals for the development of efficient fault detection and diagnosis systems. Let us consider $C_{0}\lceil n\rceil$ as the original signal sequence, whereas after the convolution with $h$ and $g$ quadrature mirror filters, the signal

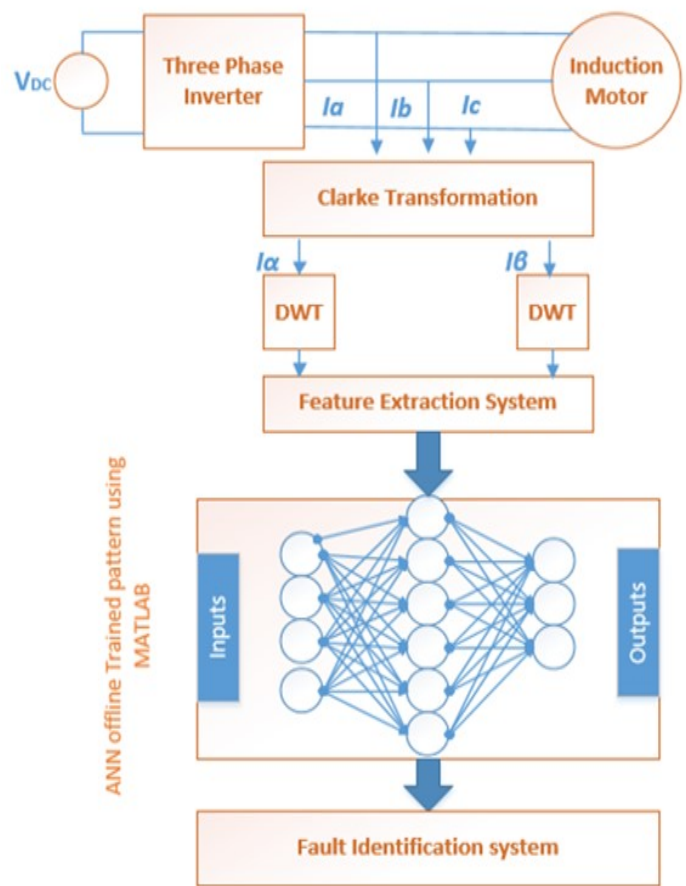

Figure 1. Structure of fault detection and diagnosis system.

sequence can be decomposed into approximation and detail components $C_{1}\lceil n\rceil$ and $d_{1}\lceil n\rceil$ at scale 1 . After that, the approximation component $C_{1}\lceil n\rceil$ can be further decomposed into $C_{2}\lceil n\rceil$ and $D_{2}\lceil n\rceil$ at the next scale and so on. Mathematically it can be represented as,

$$
\begin{aligned}
& c_{m}\lceil n\rceil \sum_{k} h\lceil k-2 n\rceil c_{m-1}\lceil k\rceil, \\
& d_{m}\lceil n\rceil=\sum_{k} g\lceil k-2 n\rceil c_{m-1}\lceil k\rceil,
\end{aligned}
$$

where $m, n$, and $k$ represent the scale of decomposition, sampling points, and translation coefficients, respectively.

Daubechies 6 wavelet is used in the current analysis. An output Clarke transformed signal is decomposed into to two levels. The DWT structure is shown in Figure 3.

The main task of analyzing current signals to detect faulty switches is performed in a feature extraction system. As mentioned before, transformation in a stationary frame is performed and DWT is implemented to extract various features from the current patterns, such as the mean of the signal and energy content for each detail and approximate signal.

Faulty switches can be detected and identified using previously obtained features. The output of this step will be 1 or 0 , in which 0 represents a normal condition and 1 represents a fault 


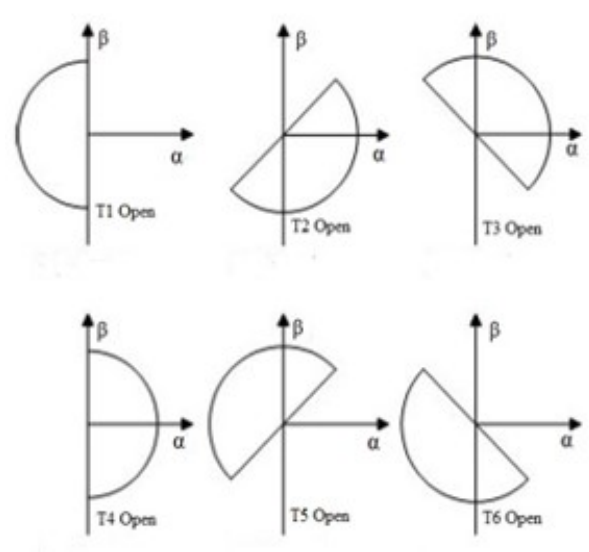

Figure 2. Current patterns in faulty modes.

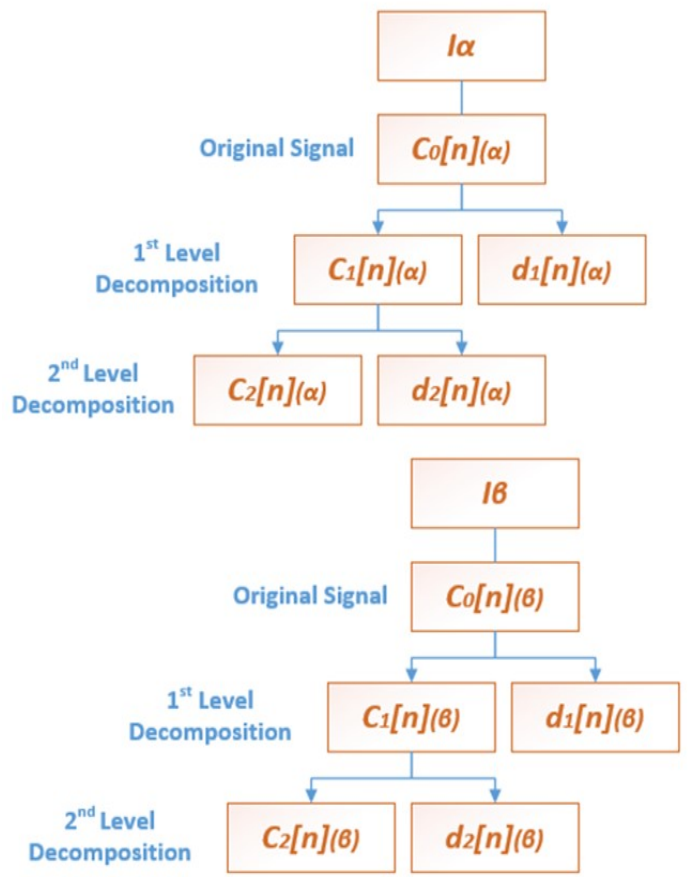

Figure 3. DWT structure.

in the respective switch. Clarke transformed $(\alpha-\beta)$ current signals were decomposed using the 2 level Daubechies 6 wavelet decomposition technique, and the mean values of the signal and energy content of each original and detail components are calculated using the following equations:

$$
\begin{aligned}
& \text { Mean } c_{2}(\alpha)=\frac{1}{\text { length } c_{2}} \sum_{n=0}^{N} c_{2}\lceil n\rceil, \\
& \text { Mean } d_{2}(\alpha)=\frac{1}{\text { length } d_{2}} \sum_{n=0}^{N} d_{2}\lceil n\rceil,
\end{aligned}
$$

$$
\text { Mean } d_{1}(\alpha)=\frac{1}{\text { length } d_{1}} \sum_{n=0}^{N} d_{1}\lceil n\rceil \text {. }
$$

Extracted features after implementing DWT on $I_{\beta}$ :

$$
\begin{aligned}
& \text { Mean } c_{2}(\beta)=\frac{1}{\text { length } c_{2}} \sum_{n=0}^{N} c_{2}\lceil n\rceil, \\
& \text { Mean } d_{2}(\beta)=\frac{1}{\text { length } d_{2}} \sum_{n=0}^{N} d_{2}\lceil n\rceil, \\
& \text { Mean } d_{1}(\beta)=\frac{1}{\text { length } d_{1}} \sum_{n=0}^{N} d_{1}\lceil n\rceil .
\end{aligned}
$$

In these equations, ' $n$ ' defines the number of samples. Content of each detail signal of DWT for both $I \alpha$ and $I \beta$ is calculated using the equations below, and these values were used as a feature vector to detect and identify the switching faults. Energy content of the detail signal at each level $j$ is given by the following equations:

$$
\begin{aligned}
& E_{j}=\sum_{i=1}^{J}\left|D_{j}(k)\right|^{2} \\
& E_{t o t}=\sum_{j} \sum_{k}\left|D_{j}(k)\right|^{2}=\sum_{j} E_{j} .
\end{aligned}
$$

\subsection{Neural Network System}

Biologically inspired neural models are the base of artificial neural networks. These systems are normally used to estimate functions that generally depend on a large number of unknown inputs, as shown in Figure 4.

A feed forward network-based artificial neural network (ANN) is used as the input data contained continuous features. The proposed ANN is based on one input layer with 12 neurons, one hidden layer, and one output layer with 13 neurons corresponding to the number of faults we want to detect. The target output of the system was 1 . The target output for a normal case would be as follows:

$$
\text { Target Output }=\left[\begin{array}{lllllllllllll}
1 & 0 & 0 & 0 & 0 & 0 & 0 & 0 & 0 & 0 & 0 & 0 & 0
\end{array}\right],
$$

where 1 represents the normal condition and 0 represents that, currently, there is no fault. For fault t1, the output will be as follows:

$$
\text { Target Output }=\left[\begin{array}{lllllllllllll}
0 & 1 & 0 & 0 & 0 & 0 & 0 & 0 & 0 & 0 & 0 & 0 & 0
\end{array}\right] .
$$




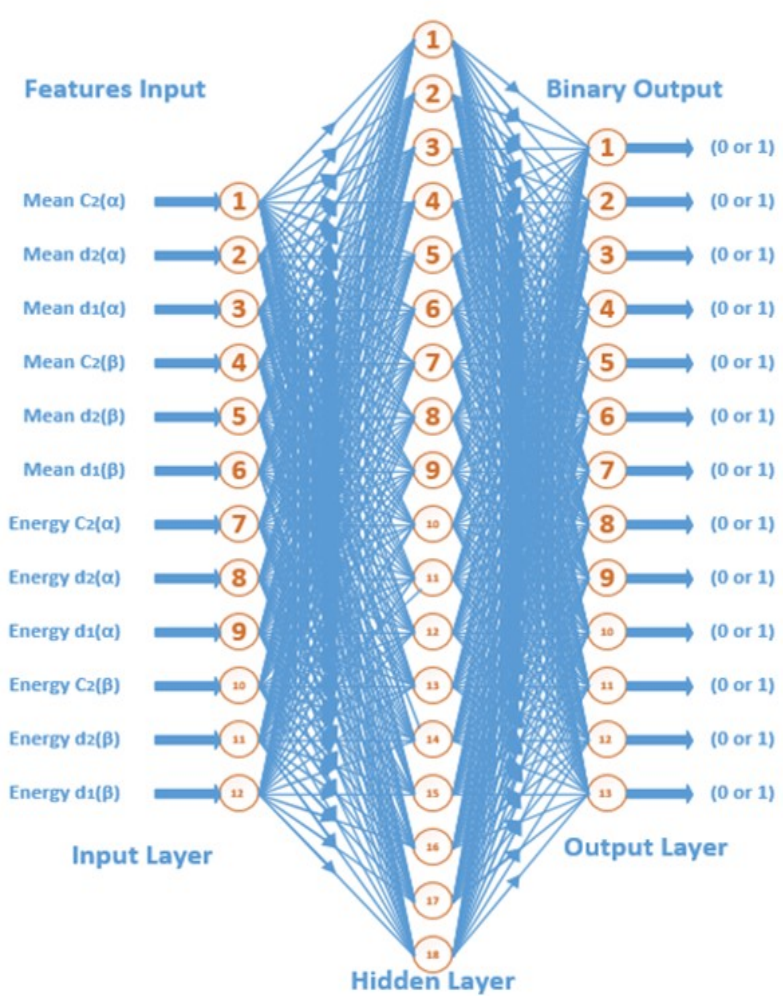

Figure 4. Architecture of an artificial neural network.

\section{Simulation Studies}

The MATLAB/Simulink simulation study is examined in this section to confirm the proposed system reliability. The designed system is tested in cases of single and multiple faults at a time. Figure 5 shows the basic model used for the analysis of stator current of a three-phase inverter-fed induction motor. The three phase induction motor was operated by an IGBT inverter using a speed control drive system, and three-phase stator current signals are extracted along with the Clarke transformed $(\alpha-\beta)$ current signals.

In the simulation test sets, the system demonstrated satisfactory classification performance in both single and multiple faults cases. Figure 6 shows the DWT block used to implement a discrete wavelet transform on the Clarke transformed stator current. The extracted $(\alpha-\beta)$ current from the motor drive system was processed through the DWT in order to decompose and extract information from the original current signals. The 2 level Daubechies 6 wavelet decomposition technique was performed on the original current signals.

Figure 7 shows the feature extraction and neural network implementation for fault identification. The decomposed signal obtained after the DWT is used for feature extraction. MATLAB

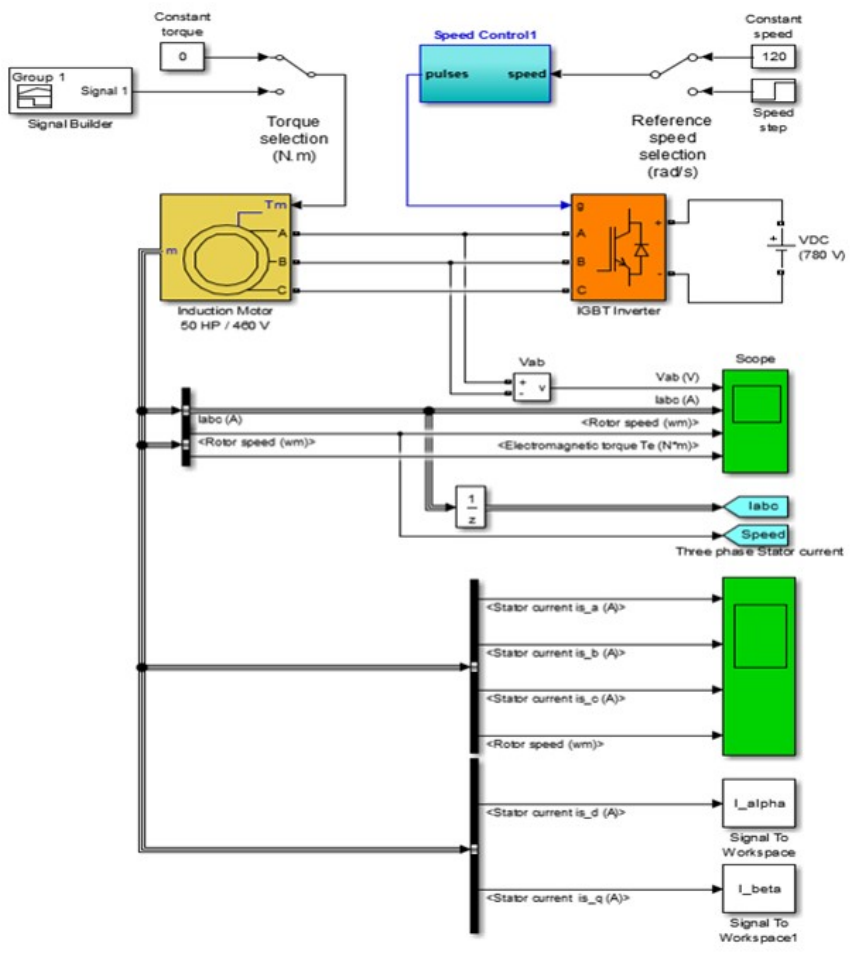

Figure 5. Neural network based fault detection and diagnosis system.

functions were used to extract features like mean of the signals and energy content from each decomposed wavelet. These features form a feature vector that is fed to the neural network to distinguish the faults.

Figure 8 shows the Clark transformed output current pattern in a normal case, Figure 9 shows the Clark transformed output current pattern for single switching faults, and Figure 10 shows the Clark transformed output current pattern for multiple switching faults. The fault detection and diagnosis system output results for single and multiple faults can be seen in Figure 11.

The designed system's simulation output results in Figure 11 show that the system works efficiently in both single and multiple switch fault modes. Simulink based system detects single and multiple faults with $100 \%$ accuracy.

\section{Conclusion}

This study presented a system for faulty switch detection and diagnosis in a three-phase inverter connected with an induction motor. The features of the output current waveform were investigated by simulation. Important features such as energy content and mean of the signal were obtained by using discrete wavelet transform. A built-in function nftool in MATLAB was used 


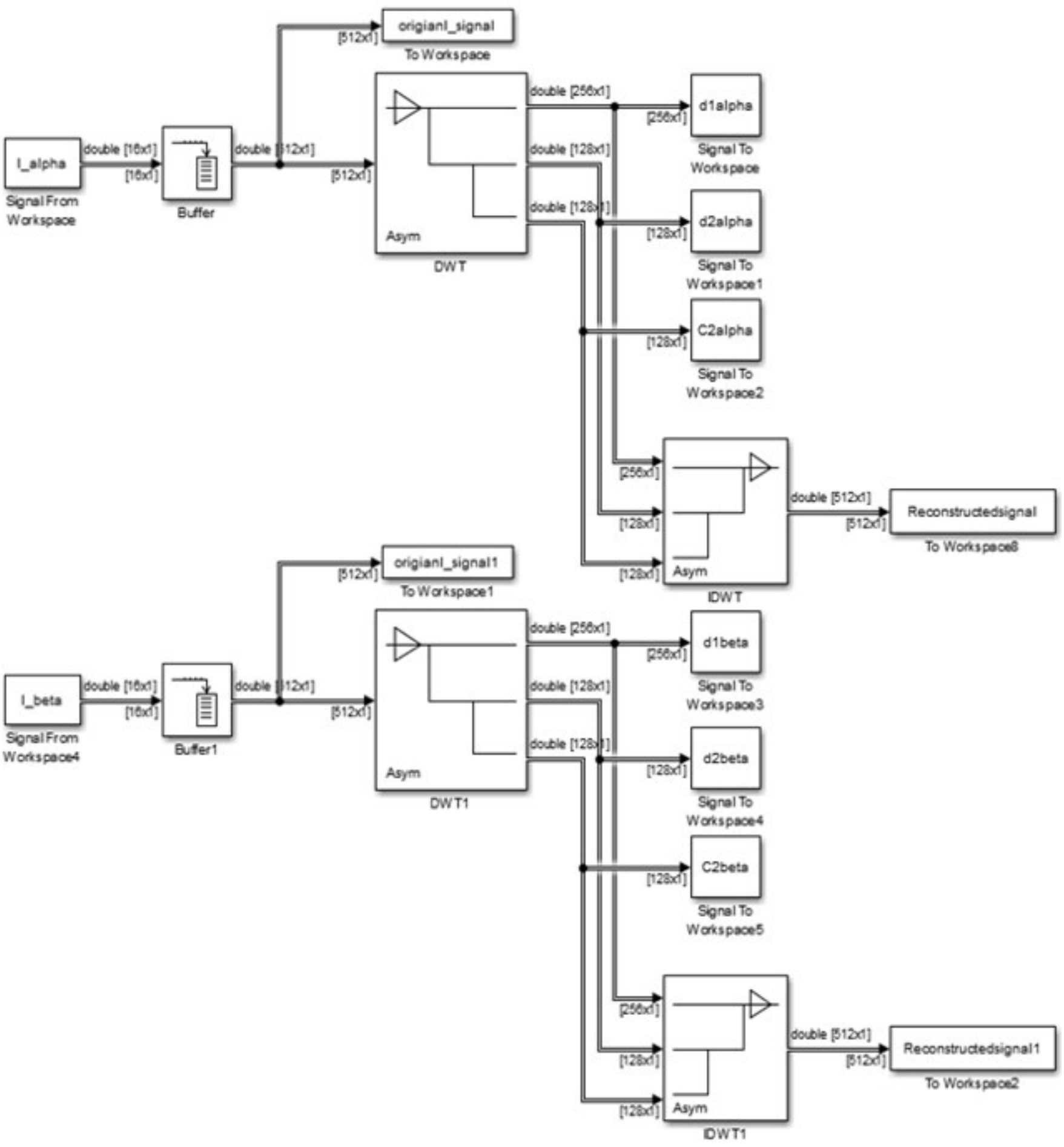

Figure 6. DWT implementation.

to perform offline training of the neural network. Extracted features play a vital role in fault detection and localization. Comparing with previous fault detection techniques, most of them cannot detect multiple faults with such high accuracy.
Furthermore, those diagnostic techniques require complex calculations. No additional sensors and complicated calculations are required for the designed system. Additionally, faulty switch detection and identification can be performed in every single 


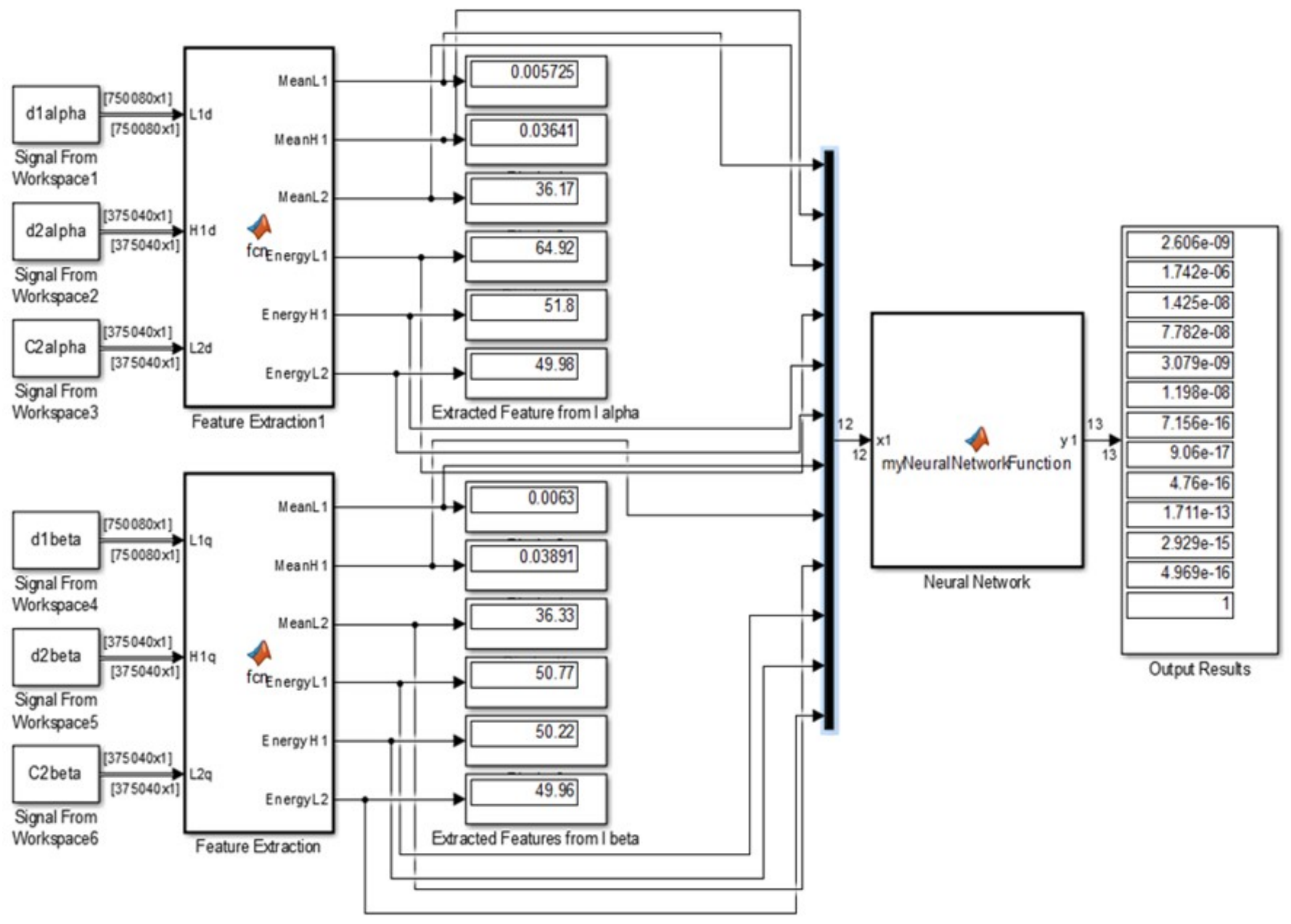

Figure 7. Feature extraction and a neural network for fault identification.

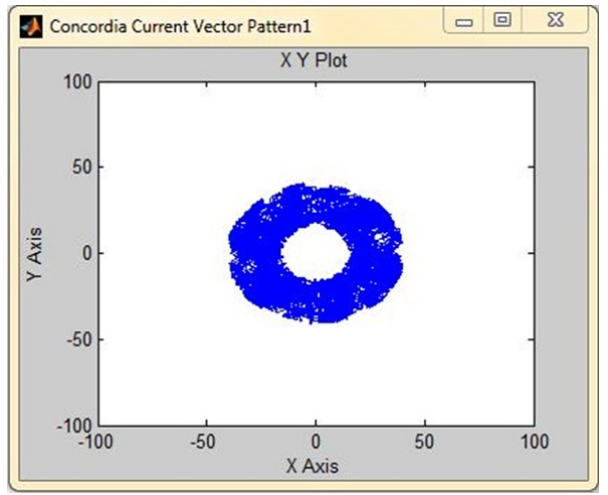

Figure 8. $\alpha-\beta$ transformed current pattern graph for the system in normal mode.

current cycle, which proves that system performance is much faster and more accurate than any prior systems as they take two or more cycles for fault detection.

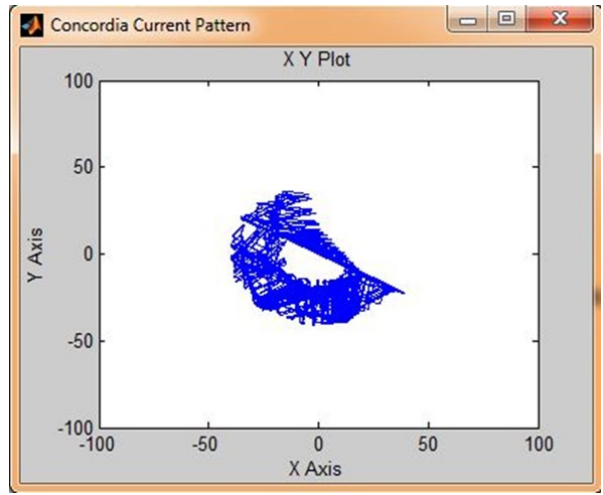

Figure 9. $\alpha-\beta$ transformed current pattern graph for the system in single fault mode (S3).

\section{Conflict of Interest}

No potential conflict of interest relevant to this article was reported. 


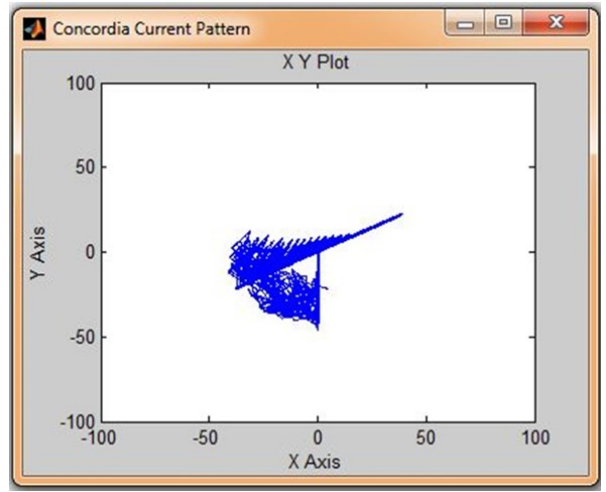

Figure 10. $\alpha-\beta$ transformed current pattern graph for the system in double fault mode (S3\&S4).

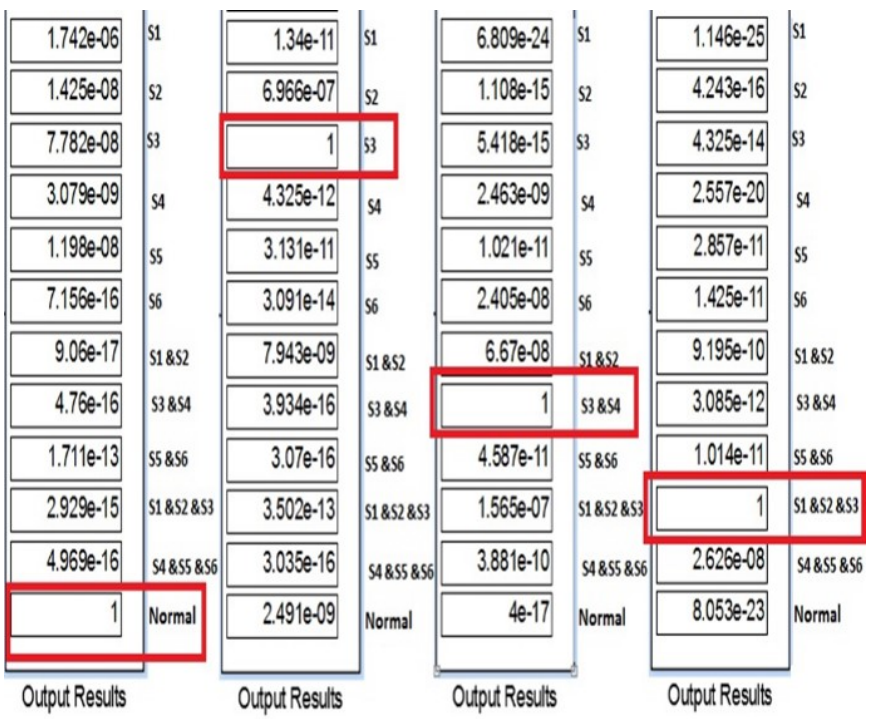

Figure 11. Simulation output results in various modes: (a) normal, (b) single fault, (c) double fault, (d) triple fault.

\section{Acknowledgements}

This work (Grants No.C0268141) was supported by Business for Academic-industrial Cooperative establishments funded Korea Small and Medium Business Administration in 2015.

\section{References}

[1] D. Diallo, M. E. H. Benbouzid, D. Hamad, and X. Pierre, "Fault detection and diagnosis in an induction Machine drive: a pattern recognition approach based on concordia stator mean current vector," IEEE Transactions on Energy Conversion, vol. 20, no. 3, pp. 512-519, 2005. http://doi. org/10.1109/TEC.2005.847961
[2] J. O. Estima and A. J. Marques Cardoso, "A new algorithm for real-time multiple open-circuit fault diagnosis in voltage-fed PWM motor drives by the reference current errors," IEEE Transactions on Industrial Electronics, vol. 60, no. 8, pp. 3496-3505, 2013. http: //doi.org/10.1109/TIE.2012.2188877

[3] P. Lezana, R. Aguilera, and J. Rodriguez, "Fault detection on multicell converter based on output voltage frequency analysis," IEEE Transactions on Industrial Electronics, vol. 56, no. 6, pp. 2275-2283, 2009. http://doi.org/10.1109/ TIE.2009.2013845

[4] M. Sivakumar, R. M. S. Parvathi, and S. Chandrasekar, "Diagnostic study of short-switch fault of cascaded Hbridge multilevel inverter using discrete wavelet transform and neural networks," International Journal of Applied Engineering Research, vol. 9, no. 21, pp. 10087-10106, 2014.

[5] S. Khomfoi and L. M. Tolbert, "Fault diagnostic system for a multilevel inverter using a neural network," IEEE Transactions on Power Electronics, vol. 22, no. 3, pp. 1062-1069, 2007. http://doi.org/10.1109/TPEL.2007. 897128

[6] R. Sarathi, S. Chandrasekar, C. Venkataseshaiah, and N. Yoshimura, "Condition monitoring of outdoor polymeric insulation structures using wavelets and neural networks," in Proceedings of 2003 Annual Report Conference on Electrical Insulation and Dielectric Phenomena, Albuquerque, NM, 2003, pp. 398-401. http://doi.org/10.1109/ CEIDP.2003.1254877

[7] S. Chandrasekar, C. Kalaivanan, A. Cavallini, and G. C. Montanari, "Investigations on leakage current and phase angle characteristics of porcelain and polymeric insulator under contaminated conditions," IEEE Transactions on Dielectrics and Electrical Insulation, vol. 16, no. 2, pp. 574 583, 2009. http://doi.org/10.1109/TDEI.2009.4815193

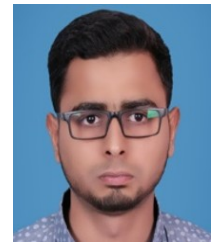

Ali Rohan received the B.S. degree in Electrical Engineering from the University of Faisalabad in 2012, and is currently an M.S. student in Kunsan National University. His research interests include renewable energy systems, power electronics, fuzzy logic, neural network, and intelligent control systems. 
Phone: +82-10-2857-6080

E-mail: ali_rohan2003@hotmail.com

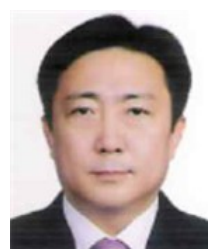

Sung Ho Kim received the M.S. and Ph.D. degrees in Electrical Engineering from Korea University, Seoul, in 1986 and 1991, respectivley. He was a postdoctoral researcher in Hiroshima University, Japan, in 1996. He is a professor at Kunsan National University from 1991. His research interests include wind turbine system, sensor networks, neural network, fuzzy logic, and intelligent control systems.

Phone: +82-10-2610-1224

E-mail: shkim@kunsan.ac.kr 